\title{
In vitro assay system for primordial germ cell development
}

\author{
Ikuma Maeda $^{1}$, Yasuhisa Matsui ${ }^{1}$ \\ ${ }^{I}$ Cell Resource Center for Biomedical Research, Institute of Development, Aging and Cancer, Tohoku University, Sendai, Miyagi \\ 980-8575, Japan \\ Cell Research (2009) 19:1125-1126. doi:10.1038/cr.2009.115; published online 1 October 2009
}

In mammalian embryos, primordial germ cells (PGCs) are induced in proximal epiblast by BMP signals expressed in extraembryonic tissues [1], and one of the most important downstream key players is Blimp1 [2]. Blimp1 is known to act as a suppressor of somatic genes' expression in PGC specification process. It is assumed that there are still many unidentified molecules involved in PGC development. However, to analyze gene functions in mammalian embryos by generating knockout animals is labor-intensive, and a more concise way to efficiently identify genes regulating PGC development is desirable.

It has been shown that PGC like cells can be obtained from the embryonic stem (ES) cell differentiation system [3]. Because the process of in vitro $\mathrm{PGC}$ differentiation is expected to reflect some aspects of PGC determination mechanism in vivo, it is reasonable to use the differentiation system as a tool for analysis of PGC development. In a study recently published in Nature [4], West and colleagues utilized ES cell differentiation system as an in vitro assay system and found that a gene called Lin28 was required for PGC development.

The authors' group formerly re-

Correspondence: Ikuma Maeda ${ }^{\mathrm{a}}$, Yasuhisa Matsui $^{\mathrm{b}}$

Tel: +81-22-717-8571; Fax: +81-22-717-8573

aE-mail: ikuma@idac.tohoku.ac.jp

bE-mail: ymatsui@idac.tohoku.ac.jp ported that PGC-like cells could be obtained from embryoid bodies (EBs) derived from mouse ES cells [5]. West and colleagues improved their previous system by utilizing Stella-GFP as a selection marker [6]. Stella is known to be the earliest marker of specified PGCs in mouse embryos, but it is also expressed in inner cell mass (ICM) of blastocyst and undifferentiated mouse ES cells. It was recently revealed that Stella expression in undifferentiated ES cells did not necessarily indicate that the cells had germ cell properties, but rather reflected that a portion of ES cells still retained ICM properties [7]. Once ES cells differentiated to EBs, Stella expression disappeared almost in all parts of EBs and only a small portion of cells keep the expression. The authors showed that EB-derived Stella-GFP positive cells had germ cell properties by the following findings. First, loss of genomic imprinting, a hallmark of germ cell differentiation, was observed in the Stella-GFP positive cells. Second, many of germline and meiosis marker genes, such as Blimp1, Dazl and Boule, were highly enriched in the cells. In addition, a microarray analysis revealed that these cells had a similar gene expression profile to that of embryo-derived PGCs. However, functionality of the cells as germ cell lineage has not been examined.

Next, the authors employed RNAmediated interference (RNAi) to identify genes involved in germ cell development. Thirty candidate genes were selected based on microarray analyses by the authors and the other group, and they were subjected to RNAi in ES cells by the lentiviral infection and then tested whether the knockdown cells retained an ability to differentiate into PGC-like cells. They found that knockdown against one of the candidate genes, namely Lin28, resulted in failure of the ES cells to differentiate into PGC-like cells. Lin28 has been known to inhibit maturation of let-7 miRNA, and it has been suggested that the gene is closely related to pluripotency [8]. Lin28-knockdown ES cells could produce Stella-GFP positive cells in EBs, but they failed to form tissue-nonspecific alkaline-phosphatase (TNAP) positive EG colonies, an another hallmark of germ cells, and the erasure of genomic imprinting was abolished in these cells. On the contrary, forced expression of Lin28 in ES cells by tetracycline-induced system resulted in upregulation of germ cell markers in EB differentiation and increase of EB-derived TNAP $^{+}$EG colony number. Therefore, Lin 28 can act both positively and negatively for PGC formation in vitro. The authors showed that Lin28 expressed semispecifically in Stella ${ }^{+}$PGCs at least in PGC surrounding region of early embryo (E7.5). In later stage (E12.5), Lin28 expression in PGCs gradually declined but remained in some extent.

It was revealed that $\operatorname{Lin} 28$ was required for in vitro derivation of PGC- 
like cells. Then, is this also true for PGC development in vivo? To answer this question, the authors made chimera embryos using the Lin 28 knockdown Stella-GFP ES cells and tested their germline contribution. If Lin 28 is required for germline development, Stella-GFP ${ }^{+}$germ cell number should be reduced. As a result, the knockdown ES cells failed to contribute to germ cells, whereas chimerism of other tissues was not affected. In addition, ectopic expression of $\operatorname{Lin} 28$ in ES cells resulted in higher contribution to germline than that of negative control ES cells.

Interestingly, a recent research showed that let-7 targeted the $3^{\prime} \mathrm{UTR}$ of Blimp1 and blocked its translation [9]. The authors assumed that Lin28 functioned in PGC development through regulation of Blimp1. Indeed, they found that ectopic expression of the 3'UTR deleted Blimp1 could rescue germline contribution of Lin28-knockdown ES cells. Likewise, Blimp1 knockdown suppressed the enhancement of germline contribution by the ectopic Lin 28 expression. Taken together, West and colleagues proposed a model that Lin 28 acted through suppression of let-7, which inhibited expression of Blimp1, then enabled Blimp1-mediated germ cell determination.

Finally, the authors showed a relationship between Lin28 and malignant tumor formation. Teratoma formation analysis revealed that Lin28overexpressed ES cell tended to form malignant tumors, whereas Lin28knockdown ES cells formed reduced size teratoma. In addition, they found that expression level of Lin 28 was consistently high in human malignant germ cell tumors but was low in benign tumors.

The authors cleverly circumvented the time-consuming process - knockout mice analysis - by combining the in vitro assay system and the chimera experiment. In fact, they succeeded in finding a gene involved in PGC development. However, it should be pointed out that their in vitro assay system may not applicable to high-throughput screening to functionally identify genes critical for PGC development at this point, because the assessment method for PGC derivation appears not to be suitable for a large number of samples. Additional improvement will be required to overcome this limitation.

It has been shown that $\operatorname{Lin} 28$ is one of the four factors which can transform human somatic cells into iPS cells [10], though it can be replaced with c-Myc. Intriguingly, our recent study revealed that another iPS related pluripotency factor Oct3/4 was also required for germline specification [11]. Taken together, there may be a common rule that pluripotency factors also have important roles in specification of PGCs.

\section{References}

1 Lawson KA, Dunn NR, Roelen BA, et al. Bmp4 is required for the generation of primordial germ cells in the mouse embryo. Genes Dev 1999; 13:424-436.

2 Ohinata Y, Payer B, O'Carroll D, et al.
Blimp1 is a critical determinant of the germ cell lineage in mice. Nature 2005; 436:207-213.

3 Marques-Mari AI, Lacham-Kaplan O, Medrano JV, Pellicer A, Simón C. Differentiation of germ cells and gametes from stem cells. Hum Reprod Update 2009; 15:379-390.

4 West JA, Viswanathan SR, Yabuuchi A, et al. A role for Lin28 in primordial germ-cell development and germ-cell malignancy. Nature 2009; 460:909913.

5 Geijsen N, Horoschak M, Kim K, Gribnau J, Eggan K, Daley GQ. Derivation of embryonic germ cells and male gametes from embryonic stem cells. Nature 2004; 427:148-154.

6 Payer B, Chuva de Sousa Lopes SM, Barton SC, Lee C, Saitou M, Surani MA. Generation of stella-GFP transgenic mice: a novel tool to study germ cell development. Genesis 2006; 44:7583.

7 Hayashi K, Lopes SM, Tang F, Surani MA. Dynamic equilibrium and heterogeneity of mouse pluripotent stem cells with distinct functional and epigenetic states. Cell Stem Cell 2008; 3:391-401.

8 Büssing I, Slack FJ, Grosshans H. Let7 microRNAs in development, stem cells and cancer. Trends Mol Med 2008; 14:400-409.

9 Nie K, Gomez M, Landgraf P, et al. MicroRNA-mediated down-regulation of PRDM1/Blimp-1 in Hodgkin/ReedSternberg cells: a potential pathogenetic lesion in Hodgkin lymphomas. Am J Pathol 2008; 173:242-252.

$10 \mathrm{Yu}$ J, Vodyanik MA, Smuga-Otto K, et al. Induced Pluripotent stem cell lines derived from human somatic cells. Science 2007; 318:1917-1920.

11 Okamura D, Tokitake Y, Niwa H, Matsui Y. Requirement of Oct3/4 function for germ cell specification. Dev Biol 2008; 317:576-584. 